\title{
Nanoscale spin reversal by nonlocal angular momentum transfer following ultrafast laser excitation in ferrimagnetic GdFeCo
}

C.E. Graves ${ }^{1,2+}$, A.H. Reid ${ }^{1,3+}$, T. Wang ${ }^{1,4}$, B. Wu ${ }^{1,2}$, S. de Jong ${ }^{5}$, K. Vahaplar ${ }^{3}$, I. Radu ${ }^{3}$, D.P. Bernstein $^{1,2}$, M. Messerschmidt ${ }^{5}$, L. Müller ${ }^{6}$, R. Coffee ${ }^{5}$, M. Bionta ${ }^{5}$, S.W. Epp ${ }^{7}$, R. Hartmann ${ }^{8}$, N. Kimmel ${ }^{9}$, G. Hauser ${ }^{9}$, A. Hartmann ${ }^{8}$, P. Holl ${ }^{8}$, H. Gorke $^{10}$, J. H. Mentink ${ }^{3}$, A. Tsukamoto ${ }^{11}$, A. Fognini $^{12}$, J.J. Turner ${ }^{5}$, W.F. Schlotter ${ }^{5}$, D. Rolles ${ }^{7}$, H. Soltau ${ }^{8}$, L. Strüder ${ }^{9}$, Y. Acremann ${ }^{12}$, A.V. Kimel $^{3}$, A. Kirilyuk ${ }^{3}$, Th. Rasing ${ }^{3}$, J. Stöhr ${ }^{5}$, A.O. Scherz ${ }^{1}$, H.A. Dürr ${ }^{1 *}$

${ }^{1}$ Stanford Institute for Materials \& Energy Sciences, SLAC National Accelerator Laboratory, 2575 Sand Hill Road, Menlo Park, CA 94025, USA, ${ }^{2}$ Department of Applied Physics, Stanford University, Stanford, CA 94305, USA, ${ }^{3}$ Radboud University Nijmegen, Institute for Molecules and Materials, Heyendaalseweg 135, 6525 AJ Nijmegen, The Netherlands, ${ }^{4}$ Department of Materials Science and Engineering, Stanford University, Stanford, CA 94305, USA, ${ }^{5}$ Linac Coherent Light Source, SLAC National Accelerator Laboratory, 2575 Sand Hill Road, Menlo Park, CA 94025, USA, ${ }^{6}$ DESY Notkestr. 85, 22607 Hamburg, Germany, ${ }^{7}$ Advanced Study Group at CFEL, Notkestr. 85, 22607 Hamburg, Germany, ${ }^{8}$ PNSensor, Otto-Hahn-Ring 6, 81739 München, Germany, ${ }^{9}$ Max Planck Institute for Extraterrestrial Physics, Giessenbachstr., 85741 Garching, Germany, ${ }^{10}$ Institute ZEL, Research Center Jülich, 52425 Jülich, Germany, ${ }^{11}$ Dept. of Electronics \& Computer Science, Nihon University, 7-24-1 Narashino-dai Funabashi, Chiba 274-8501, Japan, ${ }^{12}$ ETH Zürich, Laboratory for Solid State Physics, 8093 Zürich, Switzerland ${ }^{+}$Authors contributed equally

*Corresponding author: email hdurr@slac.stanford.edu

Ultrafast laser techniques have revealed extraordinary spin dynamics in magnetic materials (1-6) that equilibrium descriptions of magnetism (7) cannot explain. Particularly important for future applications is understanding non-equilibrium spin dynamics following laser excitation on the nanoscale, yet the limited spatial resolution of optical laser techniques has impeded such nanoscale studies. Here we present ultrafast diffraction experiments with an $x$-ray laser that probe the nanoscale spin dynamics following optical laser excitation in the ferrimagnetic alloy GdFeCo, which exhibits macroscopic all-optical switching $(4,8,9)$. Our study reveals that GdFeCo exhibits nanoscale chemical and magnetic inhomogeneities that affect the spin dynamics. In particular, we observe Gd spin reversal in Gd-rich nanoregions within the first picosecond driven by the nonlocal transfer of angular momentum from larger adjacent $\mathrm{Fe}$-rich nanoregions. These results suggest that a magnetic material's microstructure can be engineered to control transient laser-excited spins, potentially allowing faster ( 1 ps) spin reversal than in current technologies (10-12).

Nanoscale magnetization reversal provides a basis for advanced information technologies, and identifying deterministic switching mechanisms and speed limits is a key challenge. Current technologies driving spin reversal with magnetic fields or voltage-driven spinpolarized currents are limited to timescales of $\sim 100 \mathrm{ps}(10-12)$. This speed limit makes spin reversal by femtosecond (fs) optical laser pulses appealing, but in ferromagnets laser excitation typically induces loss of magnetic order (1,13-16). However, upon laser excitation the ferrimagnetic alloy GdFeCo exhibits macroscopic magnetization reversal $(4,8,9)$. This "all- 
optical switching" via inter-sublattice angular momentum transfer $(9,17)$ suggests attractive technological applications if more suitable materials can be engineered to nanoscale dimensions. Whereas an equilibrium understanding of magnetism can explain switching driven by magnetic fields or voltage-driven currents (10-12), it is insufficient to describe laser-excited spin dynamics as the electronic and spin systems are driven quickly into highly non-equilibrium states. In particular, it is critical to understand non-equilibrium spin dynamics on the nanometer (nm) lengthscale where magnetic order emerges. However, the nanoscale spin dynamics and shortrange transfer of angular momentum by optically excited electrons (18-24) within the nonequilibrium state of $\mathrm{GdFeCo}$ has remained unexplored.

Here we report x-ray laser diffraction measurements that probe the optically excited nonequilibrium spin dynamics on nm length- and fs timescales in GdFeCo (see Fig. 1a).

Additionally, we show that amorphous GdFeCo displays chemical and magnetic structure at $\sim 10 \mathrm{~nm}$. We observe the nucleation and transient existence of nm-size regions of $\mathrm{Gd}$ spin reversal, before their decay after several ps by thermal fluctuations. This temporally and spatially-resolved spin reversal is distinct from the macroscopic magnetization reversal measured previously in this material with spatial-averaging techniques $(4,8,9)$. Our results reveal a nanoscale flow of angular momentum from Fe spins originating in larger Fe-rich nanoregions to smaller Gd-rich nanoregions. We focus on this nonlocal transfer by using high optical pump fluences that maximize spin transport while causing overall demagnetization of the sample. Additionally, the nonlocal transfer is highlighted by use of x-ray diffraction, which is spatially sensitive to nanoscale spin variations. We find that nonlocal angular momentum transfer between $\mathrm{Fe} 3 \mathrm{~d}$ and $\mathrm{Gd} 4 \mathrm{f}$ spins following laser excitation causes $\mathrm{Gd}$ spin reversal in Gd-rich nanoregions where this transfer is seemingly amplified.

The macroscopic magnetic state of our sample $\mathrm{Gd}_{24} \mathrm{Fe}_{66.5} \mathrm{Co}_{9.5}$ originates from two distinct sub-lattices of spins that are antiferromagnetically coupled. The transition metals (Fe and Co) provide one sub-lattice, which consists of both atomic $3 \mathrm{~d}$ and mobile s-p spins, while the rare earth metal Gd gives rise to a second sublattice with oppositely aligned spins mainly localized in the 4f shell. Although GdFeCo is widely considered a homogeneous amorphous alloy, we find that at nm lengthscales the material's chemical homogeneity is broken by chemical segregation. The local elemental variations for Gd, Fe and Co are shown in Fig. 1b, as measured by scanning transmission electron microscopy (STEM) with elemental sensitivity from energy dispersive $\mathrm{x}$-ray spectroscopy (EDX). This characterization yields the surprising result of nanoscale chemical inhomogeneity, and the question remains how this local structure affects the spin sub-systems. We address this question using soft x-ray scattering, and therefore shift from mapping real-space to probing reciprocal-space.

The $x$-ray scattering experimental setup is shown schematically in Fig. 1a. Circularly polarized x-rays selectively detect the Fe-3d and Gd-4f spins by resonantly exciting core electrons into their respective spin shell (see Methods and Supplementary Information). Incident $\mathrm{x}$-rays are transmitted non-uniformly due to local modulations of the $\mathrm{x}$-ray absorption by the chemically inhomogeneous sample (Fig. 1b). The resulting x-ray diffraction patterns consist of linear combinations of reciprocal-space Fourier amplitudes $\mathrm{C}_{\mathrm{q}}$ and $\mathrm{S}_{\mathrm{q}}$ of the sample's chemical 
and magnetic real-space distributions (23-25). Fig. 1c shows the angle-integrated intensity $\mathrm{I}_{ \pm}(\mathrm{q})$ of $\mathrm{Gd} 4 \mathrm{f}$ diffraction patterns taken with circularly polarized resonant x-rays as a function of wavevector, $\mathrm{q}$, for opposite initial magnetic states (top panel). $\mathrm{C}_{\mathrm{q}}$ and $\mathrm{S}_{\mathrm{q}}$ can be separated from these data using $I_{ \pm}(q)=\left|C_{q} \pm S_{q}\right|^{2}(26)$, and are shown in the middle and bottom panels.

$\mathrm{C}_{\mathrm{q}}$ is the Fourier amplitude of the spatial chemical distribution within the sample, with an example of the latter shown in Fig. 1c. A perfectly homogeneous sample would give an $\mathrm{I}_{ \pm}(\mathrm{q})$ signal principally at $\mathrm{q}=0$. Amplitudes in $\mathrm{C}_{\mathrm{q}}$ and $\mathrm{S}_{\mathrm{q}}$ away from $\mathrm{q}=0$ indicate the presence of variations in the real-space charge and spin distributions, as q corresponds to the inverse correlation length. $\mathrm{I}_{ \pm}(\mathrm{q})$ would simply decrease with $\mathrm{q}$, as shown by the dashed line in Fig. 1c, if film thickness changes were the only variations present in the sample. However, the $\mathrm{Gd}_{\mathrm{q}}$ (solid line) of our sample clearly deviates from this monotonous behavior, due to the Gd enriched areas shown in Fig. 1b. The $\mathrm{C}_{\mathrm{q}}$ local scattering maximum at $\sim 0.6 \mathrm{~nm}^{-1}$ corresponds to a correlation length of $\sim 10 \mathrm{~nm}$ for $\mathrm{Gd}$-rich areas, in excellent agreement with the real-space data. A similar $\mathrm{C}_{\mathrm{q}}$ distribution results from Fe chemical enrichment, peaking at a q value corresponding to a correlation length of $\sim 13 \mathrm{~nm}$ (see Supplementary Information sections 1.2, 2.2). These results show that the sample segregates into Gd-rich ( $\sim 20 \%$ of the sample) and Fe-rich nanoregions ( $\sim 40 \%$ of the sample), that are spatially anti-correlated (see Fig. 2a).

Chemical segregation into Gd-rich and Fe-rich nanoregions alters the sub-lattice spin distributions, which are measured by $S_{\mathrm{q}}$, the magnetic Fourier amplitude. $\mathrm{S}_{\mathrm{q}}$ measures the deviation of local magnetic moments from the average sub-lattice magnetization. Equilibrium distributions of $S_{q}$ are shown in the top row of Fig. $2 b$ with Gd $S_{q}$ in red and Fe $S_{q}$ in blue. The $S_{q}$ distributions of the two sub-lattices show opposite magnetization directions, as expected for a ferrimagnet (8). There are two distinct contributions to $\mathrm{S}_{\mathrm{q}}$ for both $\mathrm{Gd}$ and Fe. Film thickness variations dominate for low-q values $\left(\mathrm{q}<0.2 \mathrm{~nm}^{-1}\right)$, and the integration of this contribution is identical to the signal obtained with spatial averaging techniques $(8$, see Supplementary Information). However, magnetic variations associated with the chemical segregation of the enriched regions dominate at high-q values $\left(q>0.2 \mathrm{~nm}^{-1}\right.$, shaded). The opposite sign of $S_{q}$ in the high-q region indicates the anti-correlation of the charge and spin distributions: chemically enriched areas simultaneously display a reduction in $\mathrm{M}_{\mathrm{z}}$ of the enriched element. By modeling the high-q $S_{q}$ contributions, we obtain the reconstructed real-space magnetization $\mathrm{M}_{\mathrm{z}}$ values in the chemically enriched regions (see Methods and Supplementary Information section 2.3). $\mathrm{M}_{\mathrm{z}}$ for Gd and Fe sub-lattices are illustrated in the center column of Fig. $2 \mathrm{~b}$ as red and blue arrows. The local $\mathrm{M}_{\mathrm{z}}$ of the enriched element is reduced in both the Gd-rich and Fe-rich nanoregions as compared to the average: $\Delta \mathrm{M}_{\mathrm{z}}^{\mathrm{Gd}}$ is $-7.8 \mu_{\mathrm{B}} / \mathrm{nm}^{3}$ in Gd-rich and $\Delta \mathrm{M}_{\mathrm{z}}^{\mathrm{Fe}}$ is $-1.1 \mu_{\mathrm{B}} / \mathrm{nm}^{3}$ in Fe-rich regions. These values indicate that local changes in the $\mathrm{Gd}$ magnetization are much larger than those in the Fe magnetization.

To probe the dynamic behaviour of $\Delta \mathrm{M}_{\mathrm{z}}$ following laser excitation, we measure the $\mathrm{S}_{\mathrm{q}}$ distributions, which are proportional to $\Delta \mathrm{M}_{\mathrm{z}}$, for different time delays $\Delta \mathrm{t}$ (shown in Fig. $2 \mathrm{~b}$ ). The fs laser excitation deposits energy directly into the $3 \mathrm{~d}$ electron system, subsequently generating mobile s-p spins (18) without exciting the $4 \mathrm{f}$ spins (15). The low-q and high-q $\mathrm{S}_{\mathrm{q}}$ contributions in 
Fig. 2b display clearly different time characteristics. While the low-q $S_{\mathrm{q}}$ scattering (average sample magnetization) decays with time, the high-q $S_{q}$ contribution increases for $G d$ and changes sign for Fe. The low-q and high-q $S_{\mathrm{q}}$ behaviour is shown in more detail for smaller time delay steps in Fig. 3. The low-q $S_{\mathrm{q}}$ data in the top panel of Fig. 3a,b reflect the quenching of the average $\mathrm{Gd}$ and Fe sub-lattice magnetizations. $\mathrm{S}_{\mathrm{q}}$ for Fe and $\mathrm{Gd}$ decays with time constants of $320 \pm 50$ fs and $570 \pm 50$ fs respectively, values typical for demagnetization (16). In addition to the high-q $S_{\mathrm{q}}$ differences noted above, the bottom panel of Fig. 3a,b reveals that both high-q responses are delayed by $360 \pm 50 \mathrm{fs}$. The $\mathrm{S}_{\mathrm{q}}$ response following laser excitation is more clearly illustrated by the reconstructed local magnetization $\mathrm{M}_{\mathrm{z}}$ for $\mathrm{Fe}$ - and $\mathrm{Gd}$-rich nanoregions (see Methods and Supplementary Information), shown in Fig. 3c. In Fe-rich nanoregions, both sublattices display demagnetization, which is also observed macroscopically (see Supplementary Information). However, the Gd-rich nanoregions respond differently: $\mathrm{M}_{\mathrm{z}}^{\mathrm{Gd}}$ of the Gd-rich regions reverses sign at $\Delta \mathrm{t} \sim 1 \mathrm{ps}$. As $\mathrm{M}_{\mathrm{z}}^{\mathrm{Fe}}$ maintains its sign, a net ferromagnetic alignment of the two sublattice magnetizations occurs within the Gd-rich nanoregions after $\sim 1$ ps and persists several ps thereafter. At 2ps, the Gd spins in Gd-rich nanoregions have reversed from their initial value of $-73 \mu_{\mathrm{B}} / \mathrm{nm}^{3}$ to $+10.7 \pm 6.4 \mu_{\mathrm{B}} / \mathrm{nm}^{3}$. This angular momentum transfer is surprisingly enhanced at lower optical pump fluences: with $16 \mathrm{~mJ} / \mathrm{cm}^{2}$ pump fluence, the Gd spins in Gd-rich nanoregions show a reversed value of $+49.3 \pm 14.0 \mu_{\mathrm{B}} / \mathrm{nm}^{3}$ after $2.5 \mathrm{ps}$. As the local change $\Delta \mathrm{M}_{\mathrm{Z}}^{\mathrm{Gd}}$ is much greater than $\Delta \mathrm{M}_{\mathrm{z}}^{\mathrm{Fe}}$, nonlocal angular momentum transfer to Gd spins within Gdrich nanoegions is required.

Analysis of the high-q $S_{\mathrm{q}}$ dynamics quantifies the amount of nonlocal angular momentum transfer to the Gd-rich nanoregions. The high-q $S_{\mathrm{q}}$ measures the local difference of the sublattice magnetizations from average, or $\Delta \mathrm{M}_{\mathrm{z}}$, within the enriched regions. While $\Delta \mathrm{M}_{\mathrm{z}}{ }^{\mathrm{Gd}}$ increases with time, the $\Delta \mathrm{M}_{\mathrm{z}}^{\mathrm{Fe}}$ becomes smaller, revealing nonlocal angular momentum transfer to the Gd-rich regions' $\mathrm{M}_{\mathrm{z}}^{\mathrm{Gd}}$, since otherwise $\Delta \mathrm{M}_{\mathrm{z}}^{\mathrm{Fe}}$ would necessarily be larger. The nonlocal transfer is characterized by $\mathrm{J}_{\mathrm{S}}$, the time-evolution of the net angular momentum flow into the Gd-rich nanoregions, shown in Fig. $3 \mathrm{~d}$. $\mathrm{J}_{\mathrm{S}}$ is the rate of change of $\Delta \mathrm{M}_{\mathrm{z}}$ or $\mathrm{J}_{\mathrm{S}}=\mathrm{d} / \mathrm{dt}\left(\Delta \mathrm{M}_{\mathrm{z}}\right)_{\mathrm{Gd} \text {-rich }}=\mathrm{d} / \mathrm{dt}$ $\left(\Delta \mathrm{M}_{\mathrm{z}}^{\mathrm{Gd}}+\Delta \mathrm{M}_{\mathrm{z}}^{\mathrm{Fe}}\right)_{\mathrm{Gd}-\text { rich }}$ and is dominated by the Gd contribution since $\mathrm{d} / \mathrm{dt}\left(\Delta \mathrm{M}_{\mathrm{z}}^{\mathrm{Gd}}\right)_{\mathrm{Gd}-\text { rich }}>\mathrm{d} / \mathrm{dt}$

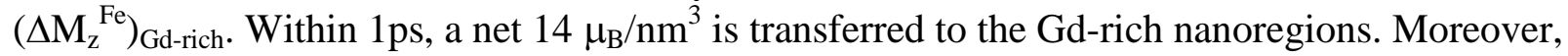
the positive sign of $\mathrm{J}_{\mathrm{S}}$ during the first ps indicates that it is the Fe spins that transport angular momentum into the Gd-rich nanoregions, as only they possess the correct sign. After $1 \mathrm{ps}, \mathrm{J}_{\mathrm{S}}$ becomes negative indicating a slower back-flow of angular momentum out of the Gd-rich regions. This backflow continues until between 4 and 6ps when the Gd magnetization switches back and the ground-state ferrimagnetism is reestablished.

It is important to consider why the nonlocal transfer of angular momentum leads to Gd spin reversal specifically within the disordered Gd-rich nanoregions. Following optical excitation, Fe $3 \mathrm{~d}$ spins are excited into s-p states $(18,21,27)$, and angular momentum then moves from Fe to various reservoirs: the local Gd reservoir, the lattice (16), and nonlocal transport $(18,19,21-24)$. Previous work on $\mathrm{GdFeCo}$ focused on the macroscopic angular momentum transfer observed at lower fluences $(8,17)$. In contrast, our measurements isolate the nanoscale spatial variations of this transfer. Additionally, we observe Gd spin reversal, not Fe, contrary to reported local 
theories of non-equilibrium dynamics $(9,17)$, and we observe the spin reversal in regions of Fe depletion where even less Fe angular momentum is available to transfer locally. Transfer of angular momentum from Fe to Gd occurs via the exchange interaction. This transfer is enhanced within the Gd-rich regions due not only to local enhancement of the $\mathrm{Gd}-\mathrm{Fe}$ exchange, but also to spin-torque scattering at the interface of the Gd-rich regions resulting from non-collinear spins and abrupt chemical variations on the electron scattering lengthscale $(28,29)$. Surprisingly, this spin transfer has a delayed onset, suggesting a threshold-like behaviour. While the observed spin transfer and reversal could be due to spin currents $(18,21,23,24)$, the presence of the delay indicates the need for further theoretical work. The spin transfer to the Gd-rich regions peaks around $\sim 1 \mathrm{ps}$, a timescale characteristic for hot electrons to reach thermal equilibrium with the lattice $(1,4,27)$. Growth of the reversed Gd-rich regions is indicated by the shift of the $\mathrm{S}_{\mathrm{q}}$ maximum to lower q values with increasing delay time, characteristic of a size increase of the spin reversed area (30). The reversed Gd magnetization dissipates following the spin transfer, proceeding via Fe spin states, as demonstrated by the fact that the increase of Fe and the decrease of $\mathrm{Gd} \Delta \mathrm{M}_{\mathrm{z}}$ have the same time constant.

To conclude, by measuring nm-fs spin scattering dynamics that are only accessible with $\mathrm{x}$ ray lasers, we demonstrate nanoscale spin reversal in GdFeCo driven by nonlocal transfer of angular momentum. Our experimental technique opens a new way to study emerging phenomena, such as order in highly non-equilibrium systems. Our results not only link two important current fields in spintronics, namely transport and ultrafast optics, but also point to the general importance of nanoscale inhomogeneities in the study of many fundamental phenomena, such as crystal growth, the role of fluctuations in high $\mathrm{T}_{\mathrm{c}}$ superconductors and the emergence of quantum electronic phases hidden in thermal equilibrium. The observed angular momentum transfer reverses spins robustly and overcomes the magnetic disorder, suggesting an effective tool for controlling laser-excited spins in microscopically engineered magnetic materials. In addition, the observed spin reversal is orders of magnitude faster than reversal by conventional voltage-driven equilibrium spin currents and may offer dramatic improvements over present spin-torque devices.

\section{Methods Summary}

The studied sample was a $30 \mathrm{~nm} \mathrm{Gd}_{24} \mathrm{Fe}_{66.5} \mathrm{Co}_{9.5}$ amorphous out-of-plane magnetized thin film deposited by magnetron sputtering on a $100 \mathrm{~nm} \mathrm{Si}_{3} \mathrm{~N}_{4}$ membrane, identical to those used in ref (8). To protect the GdFeCo layer and prevent oxidation, $5 \mathrm{~nm}$ and $10 \mathrm{~nm}$ of $\mathrm{Si}_{3} \mathrm{~N}_{4}$ were used as buffer and capping layers respectively.

Time-resolved x-ray scattering measurements were performed in transmission in a collinear pump-probe geometry. Samples were photoexcited by a $50 \mathrm{fs}$ optical laser pulse $(\lambda=800 \mathrm{~nm})$ at $16 \mathrm{~mJ} / \mathrm{cm}^{2}$ and $24 \mathrm{~mJ} / \mathrm{cm}^{2}$, and probed with 80 fs circularly polarized X-ray pulses at the Fe $\mathrm{L}_{3}$ $(707 \mathrm{eV})$ and $\mathrm{Gd} \mathrm{M}_{5}(1189 \mathrm{eV})$ edges, enabling an independent probe of $\mathrm{Gd}$ and Fe atoms. The xray pulses were passed through a thin magnetic film to produce circular polarization. Single-shot 
x-ray diffraction patterns were recorded by a high-frame-rate, low-noise X-ray pnCCD detector at the LCLS repetition rate of $60 \mathrm{~Hz}$. In between pump-probe events, the sample was reset by an external magnetic field $( \pm 0.3 \mathrm{~T})$ in alternating opposite directions. The diffracted intensity $\mathrm{I}_{+}(\mathrm{q})$ and $I_{-}(\mathrm{q})$ were recorded at fixed x-ray helicity and opposite resetting magnetic field as a function of pump-probe delay. As incident x-rays are transmitted by the sample nonuniformly due to local modulations in the sample, the resulting $\mathrm{x}$-ray diffraction pattern contains the Fourier components $\mathrm{C}_{\mathrm{q}}$ and $\mathrm{S}_{\mathrm{q}}$ of the chemical and magnetic real-space distributions respectively. The intensity of the diffracted $\mathrm{X}$-rays can therefore be written as $\mathrm{I}_{ \pm}(\mathrm{q})=\mathrm{C}_{\mathrm{q}}{ }^{2}+\mathrm{S}_{\mathrm{q}}{ }^{2} \pm 2 \mathrm{C}_{\mathrm{q}} \mathrm{S}_{\mathrm{q}}$. We analyze the transient evolution of $S_{\mathrm{q}}$ to isolate the magnetic response of the sample, which can be written as $\mathrm{S}_{\mathrm{q}}=1 / \operatorname{sqrt}(8) *\left(\mathrm{I}_{+}-\mathrm{I}_{-}\right) / \operatorname{sqrt}\left(\mathrm{I}_{+}+\mathrm{I}_{-}\right)$with some approximations (see Supplementary Information).

Elemental mapping of the nanoscale chemical variations of Gd and Fe was performed with an energy-dispersive X-ray (EDX) detector in scanning transmission electron microscopy (STEM) mode with a probe size of $\cdot \sim 1 \mathrm{~nm}$. All STEM-EDX measurements used a FEI Tecnai G2 F20 X-TWIN TEM with an EDAX SUTW (super ultra thin window) and analyzer. Measurements were performed on a $30 \mathrm{~nm} \mathrm{Gd}_{24} \mathrm{Fe}_{66.5} \mathrm{Co}_{9.5}$ sample grown on an $8 \mathrm{~nm} \mathrm{Si}_{3} \mathrm{~N}_{4} \mathrm{STEM}$ grid. The nominal and measured average concentrations were found to agree within $0.8 \%$.

Calculations of the time-evolution local variations in $\mathrm{M}_{\mathrm{Z}}$ used weight factors $\gamma_{\mathrm{Fe}}$ and $\gamma_{\mathrm{Gd}}$ for the magnetic changes in the respective $\mathrm{Fe}$ and $\mathrm{Gd}$ enriched regions derived from equilibrium data and modeling. These factors give the local change in the magnetization with respect to the sample average within these regions. The magnetization at any point can be described by $\mathrm{M}_{\mathrm{z}}(\mathrm{x}, \mathrm{y})$ $=\mathrm{M}_{\mathrm{z}}^{\text {average }}+\Delta \mathrm{M}_{\mathrm{z}}(\mathrm{x}, \mathrm{y})$. As the $\mathrm{S}_{\mathrm{q}}$ distribution at high-q is proportional to $\Delta \mathrm{M}_{\mathrm{z}}, \Delta \mathrm{M}_{\mathrm{z}}^{\mathrm{Gd}}=\gamma_{\mathrm{Gd}} * \mathrm{~S}_{\mathrm{q}}{ }^{\mathrm{Gd}}$ $\left(q>0.2 \mathrm{~nm}^{-1}\right.$ ), the size of $\mathrm{M}_{\mathrm{z}}$ in the chemically-enriched areas can be calculated from the $\mathrm{S}_{\mathrm{q}}$ data. The net spin flow into the Gd-enriched regions, $\mathrm{J}_{\mathrm{s}}$, is defined as the rate of change of $\Delta \mathrm{M}_{\mathrm{z}}$ or $J_{S}=\frac{\partial}{\partial t}\left(\Delta M_{Z}^{G d}+\Delta M_{Z}^{F e}\right)_{G d-r i c h}=\frac{\partial}{\partial t}\left(\gamma_{G d} S_{q>0.2 m^{-1}}^{G d}+\gamma_{F e} S_{q>0.2 ~ n m^{-1}}^{F e}\right)_{G d-r i c h}$ and the dominant contribution is from Gd as $\frac{\partial}{\partial t}\left(\gamma_{G d} S_{q>0.2 m^{-1}}^{G d}\right)_{G d-r i c h} \gg \frac{\partial}{\partial t}\left(\gamma_{F e} S_{q>0.2 m^{-1}}^{F e}\right)_{G d-r i c h}$. Therefore, $\mathrm{J}_{\mathrm{s}}$ (as shown in Fig. $3 \mathrm{~d}$ ) is calculated from the high-q $\mathrm{S}_{\mathrm{q}}$ data shown in the bottom panels of Fig. 3a,b. The total angular momentum transferred into the Gd-rich regions during the first ps is calculated by integrating $\mathbf{J}_{\mathrm{s}}$.

\section{References and notes:}

1. Beaurepaire, E., Merle, J.-C., Daunois, A. \& Bigot, J.-Y. Ultrafast spin dynamics in ferromagnetic nickel. Phys. Rev. Lett. 76, 4250 (1996).

2. Bigot, J.-Y., Vomir, M., \& Beaurepaire, E. Coherent ultrafast magnetism induced by femtosecond laser pulses. Nature Phys. 5, 515 (2009).

3. Ju, G. et al. Ultrafast generation of ferromagnetic order via a laser-induced phase transformation in FeRh thin films. Phys. Rev. Lett. 93, 197403 (2004). 
4. Stanciu, C. D. et al. All-optical magnetic recording with circularly polarized light. Phys. Rev. Lett. 99, 047601 (2007).

5. Kirilyuk, A., Kimel, A. \& Rasing, Th. Ultrafast optical manipulation of magnetic order. Rev. Mod. Phys. 82, 2731 (2010).

6. Guidoni, L., Beaurepaire, E. \& Bigot, J.-Y. Magneto-optics in the ultrafast regime: thermalization of spin populations in ferromagnetic films. Phys. Rev. Lett. 89, 17401 (2002).

7. Stöhr, J. \& Siegmann, H.C. Magnetism: from Fundamentals to Nanoscale Dynamics (Springer, 2006).

8. Radu, I. et al. Transient ferromagnetic-like state mediating ultrafast reversal of antiferromagnetically coupled spins. Nature 472, 205 (2011).

9. Ostler, T. et al. Ultrafast heating as a sufficient stimulus for magnetization reversal in a ferrimagnet. Nat. Commun. 3, 666 (2012).

10. Back, C.H. et al. Minimum field strength in precessional magnetization reversal. Science 285, 864 (1999).

11. Acremann, Y. et al. Time-resolved imaging of spin transfer switching: beyond the macrospin concept. Phys. Rev. Lett. 96, 217202 (2006).

12. Slonczewski, J.C. Current-driven excitation of magnetic multilayers. J. Magn. Magn. Mater. 159, L1 (1996).

13. Koopmans, B., Van Kampen, M., Kohlhepp, J.T. \& de Jonge, W.J.M. Ultrafast magnetooptics in nickel: magnetism or optics? Phys. Rev. Lett. 85, 844 (2000).

14. Stamm, C. et al. Femtosecond modification of electron localization and transfer of angular momentum in nickel. Nature Mater. 6, 740 (2007).

15. Wietstruk, M. et al. Hot-electron-driven enhancement of spin-lattice coupling in $\mathrm{Gd}$ and $\mathrm{Tb}$ 4f ferromagnets observed by femtosecond x-ray magnetic circular dichroism. Phys. Rev. Lett. 106, 127401 (2011).

16. Koopmans, B. et al. Explaining the paradoxical diversity of ultrafast laser-induced demagnetization. Nature Mater. 9, 259 (2010).

17. Mentink, J.H. et al. Ultrafast spin dynamics in multisublattice magnets. Phys. Rev. Lett. 108, 057202 (2012).

18. Battiato, M., Carva, K. \& Oppeneer, P.M. Superdiffusive spin transport as a mechanism of ultrafast demagnetization. Phys. Rev. Lett. 105, 027203 (2010).

19. Melnikov, A. et al. Ultrafast transport of laser-excited spin-polarized carriers in $\mathrm{Au} / \mathrm{Fe} / \mathrm{MgO}(001)$. Phys. Rev. Lett. 107, 076601 (2011).

20. Malinowski, G. et al. Control of speed and efficiency of ultrafast demagnetization by direct transfer of spin angular momentum. Nature Phys. 4, 855 (2008).

21. Battiato, M., Carva, K. \& Oppeneer, P.M. Theory of laser-induced ultrafast superdiffusive spin transport in layered heterostructures. Phys. Rev. B. 86, 024404 (2012).

22. Rudolf, D. et al. Ultrafast magnetization enhancement in metallic multilayers driven by superdiffusive spin current. Nat. Commun. 3, 1037 (2012). 
23. Vodungbo, B. et al. Laser-induced ultrafast demagnetization in the presence of a nanoscale magnetic domain network. Nat. Commun. 3, 999 (2012).

24. Pfau, B. et al. Ultrafast optical demagnetization manipulates nanoscale spin structure in domain walls. Nat. Commun. 3, 1100 (2012).

25. Gutt, C. et al. Single-pulse resonant magnetic scattering using a soft x-ray free-electron laser. Phys. Rev. B. 81, 100401 (2010).

26. Hannon, J.P. Trammell, G.T., Blume, M. \& Gibbs, D. X-ray resonance exchange scattering. Phys. Rev. Lett., 61, 1245 (1988).

27. Rhie, H.-S., Dürr, H.A. \& Eberhardt, W. Femtosecond electron and spin dynamics in Ni/W(110) films. Phys. Rev. Lett. 90, 247201 (2003).

28. Stiles, M.D. \& Zangwill, A. Anatomy of spin-transfer torque. Phys. Rev. B. 66, 014407 (2002).

29. Bass, J. \& Pratt, W.P. Spin diffusion lengths in metals and alloys, and spin-flipping at metal/metal interfaces: an experimentalist's critical review. J. Phys. Condens. Matter 19, 183201 (2007).

30. Zuo, J.-K., Wendelken, J.F., Dürr, H. \& Liu, C.-L. Growth and coalescence in submonolayer homoepitaxy on $\mathrm{Cu}(100)$ studied with high-resolution low-energy electron diffraction. Phys. Rev. Lett. 72, 3064 (1994).

Acknowledgments: Research at Stanford is supported by U.S. DOE, Office of Basic Energy Sciences, Materials Sciences and Engineering Division under contract DE-AC02-76SF00515. Portions of this research were carried out on the SXR Instrument at the Linac Coherent Light Source (LCLS), a division of SLAC National Accelerator Laboratory and an Office of Science user facility operated by Stanford University for the U.S. Department of Energy. The SXR Instrument is funded by a consortium whose membership includes the LCLS, Stanford University through the Stanford Institute for Materials Energy Sciences (SIMES), Lawrence Berkeley National Laboratory (LBNL), University of Hamburg through the BMBF priority program FSP 301, and the Center for Free Electron Laser Science (CFEL). Additionally, other portions of this research were carried out at the Stanford Synchrotron Radiation Lightsource, a Directorate of SLAC National Accelerator Laboratory and an Office of Science User Facility operated for the U.S. Department of Energy Office of Science by Stanford University. C.E.G. is a NSF Graduate Research Fellow. This work was also supported by the European Community's Seventh Framework Programme FP7/2007-2013 (grants NMP3-SL-2008-214469 (UltraMagnetron) and 214810 (FANTOMAS)), the European Research Council ERC Grant agreement No 257280 (Femtomagnetism), the Foundation for Fundamental Research on Matter (FOM), the Netherlands Organization for Scientific Research (NWO), the Nihon University Strategic Projects for Academic Research, the DFG grant SFB925, the Excellence cluster

"Frontiers in Quantum Photon Science" and the Max Planck Society through development and operation of the pnCCD cameras at CFEL. We are thankful to Ann Marshall for assistance and discussion on STEM-EDX measurements. 
Author contributions: I.R., A.V.K., A.K., Th.R., W.S., A.O.S, J.S., H.A.D. designed and coordinated the project; C.E.G., A.H.R., B.W., T.W., S.J., K.V., I.R., D.P.B., M.M., L.M., A.F., Y.A., H.A.D. and A.O.S. performed the x-ray diffraction measurements; W.F.S. and J.J.T. operated the SXR beamline; R.C. and M.B. operated the pump laser and synchronization; S.W.E., R.H., A.H., N.K., D.R. G.H., P.H., H.G., H.S. and L.S. provided and operated the pnCCD detector; T.W. performed the STEM measurements; C.E.G., A.H.R., S.J., H.A.D and A.O.S. performed the data analysis; A.H.R. developed the chemical distribution model; A.T. grew and optimized the samples; H.A.D., C.E.G. and A.H.R. coordinated work on the paper with contributions from A.O.S., J.S., Th.R., A.V.K., A.K., J.H.M. and discussions with all authors. 
Figures: 


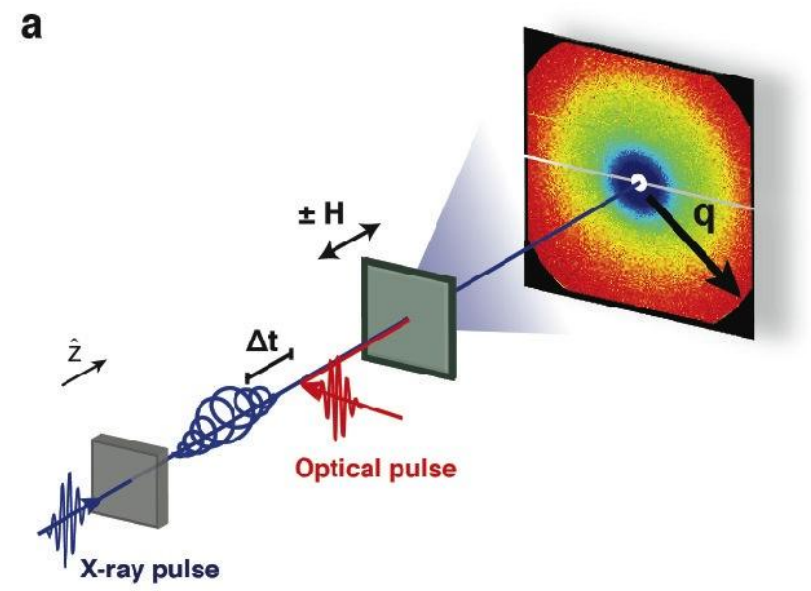

b

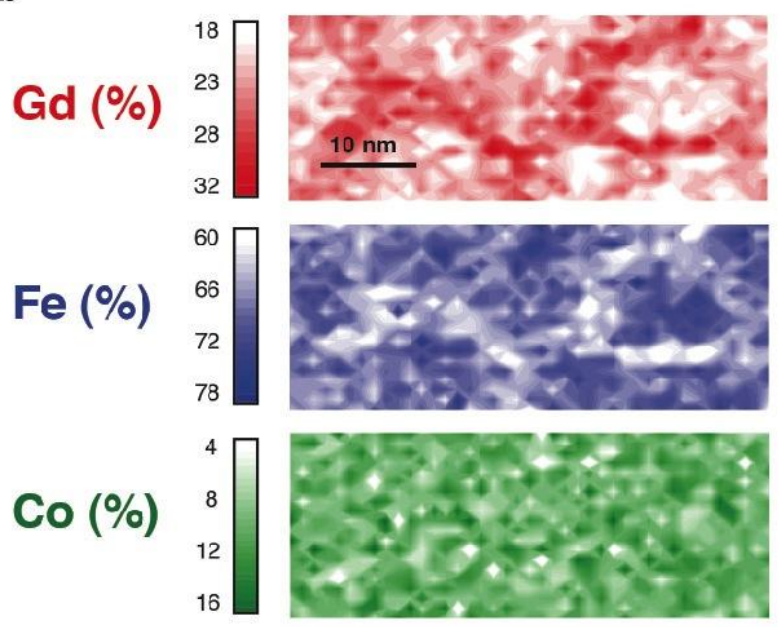

C

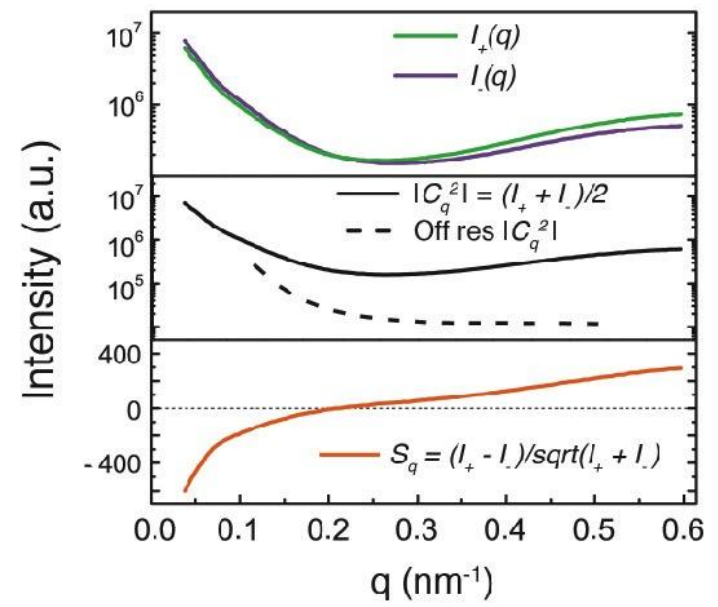

FIG. 1: Measured Chemical and Spin inhomogeneity in GdFeCo. Experimental optical-pump $\mathrm{x}$-ray probe geometry with description of measured quantities in scattering and initial nanoscale elemental variance. (a) Schematic of the magnetic x-ray diffraction experiment. The diffracted intensity is measured as a function of wavevector q, or inverse correlation length. (b) Local charge nanoscale variations for $\mathrm{Gd}$, $\mathrm{Fe}$, and $\mathrm{Co}$ in $\mathrm{Gd}_{24} \mathrm{Fe}_{66.5} \mathrm{Co}_{9.5}$ are shown from the same 
sample region as measured with STEM-EDX. Darker colored areas indicate elemental enrichment. White denotes below average concentrations. The maps show the anti-correlation of Gd-rich and Fe-rich regions and both Gd and Fe display chemically enriched areas with a frequency of $\sim 10 \mathrm{~nm}$. (c) Illustration of measured $\mathrm{I}_{ \pm}(\mathrm{q})=\mathrm{C}_{\mathrm{q}}{ }^{2}+\mathrm{S}_{\mathrm{q}}{ }^{2} \pm 2 \mathrm{C}_{\mathrm{q}} \mathrm{S}_{\mathrm{q}}$ data and the separation of $\mathrm{C}_{\mathrm{q}}$ and $\mathrm{S}_{\mathrm{q}}$ for the $\mathrm{Gd} 4 \mathrm{f}$ system. The top panel shows the angle-integration of the recorded intensity patterns for parallel and antiparallel orientations of the sample magnetization and beam propagation direction as $\mathrm{I}_{+}(\mathrm{q})$ and $\mathrm{I}_{-}(\mathrm{q})$. The charge scattering, $\mathrm{C}_{\mathrm{q}}{ }^{2}$, related to the sum of $\mathrm{I}_{ \pm}(\mathrm{q})$ (see methods) is shown in the middle panel, and shows an intensity distribution at a $\mathrm{q}$ value $\left(\sim 0.6 \mathrm{~nm}^{-1}\right)$ in line with the chemical enrichment periodicity $(\sim 10 \mathrm{~nm})$ observed in (b). We also show the $\mathrm{C}_{\mathrm{q}}{ }^{2}$ off-resonance data as a dotted line for comparison. $\mathrm{S}_{\mathrm{q}}$, the Fourier component of the spin distribution in the sample, related to the difference of $\mathrm{I}_{ \pm}(\mathrm{q})$ (see methods) is shown in the bottom panel. The intensity distribution of $S_{q}$ at $q \sim 0.6 \mathrm{~nm}^{-1}$ indicates the coexistence of spin variation with the chemically enriched regions shown in (b).

a
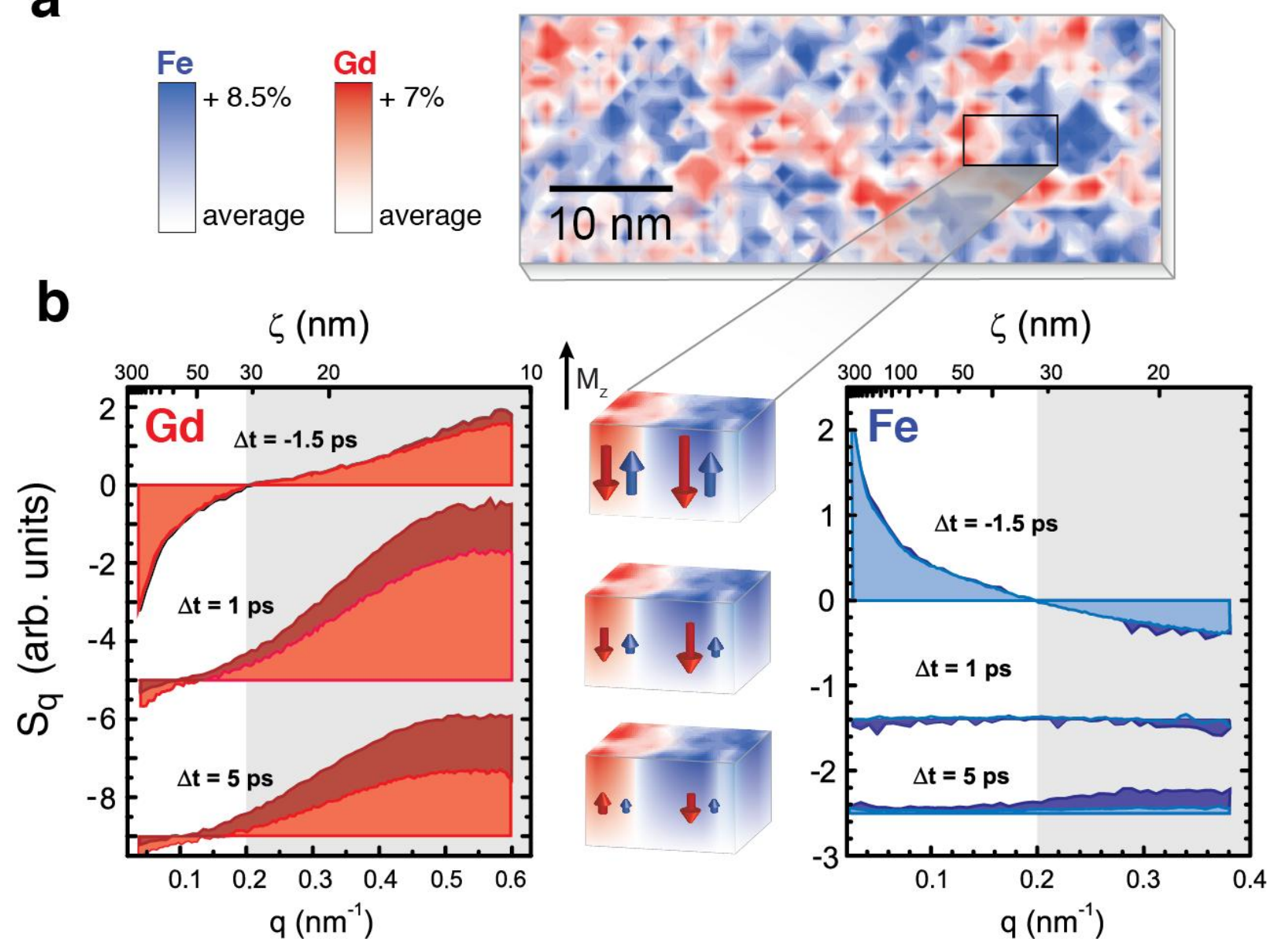
FIG. 2: Nanoscale variations in charge and spin distributions drive q-dependent magnetic scattering dynamics (a) Overlaid nanoscale elemental concentration map for Gd and Fe from taken in Fig. $1 \mathrm{~b}$ for $\mathrm{Gd}_{24} \mathrm{Fe}_{66.5} \mathrm{Co}_{9.5}$ were obtained with STEM-EDX. Red areas indicate a local enrichment of Gd atoms and blue areas indicate a local enrichment of Fe atoms, with enrichment defined as a deviation higher than $3 \%$ above average (white shading). These data in combination with the $\mathrm{x}$-ray scattering data give the average local variation in elemental concentration as $5.1 \%$ for Gd-enriched areas and $1.9 \%$ for Fe-enriched areas. Additionally, Gd-rich nanoregions cover $\sim 20 \%$ of the sample, and Fe-rich nanoregions cover $\sim 40 \%$ of the sample (see Supplementary Information). (b) Magnetic x-ray diffraction, $\mathrm{S}_{\mathrm{q}}$, of $\mathrm{Gd} 4 \mathrm{f}$ and $\mathrm{Fe} 3 \mathrm{~d}$ vs. wavevector, $\mathrm{q}$, for the indicated $\mathrm{x}$-ray delay times, $\Delta \mathrm{t}$. The darker shaded areas show data with $16 \mathrm{~mJ} / \mathrm{cm}^{2}$ and lighter shaded areas show data with $24 \mathrm{~mJ} / \mathrm{cm}^{2}$ optical pump fluence. The thermalized temperature at $1 \mathrm{ps}$ for both fluences is above $\mathrm{T}_{\mathrm{c}}$. Negative time refers to equilibrium conditions before optical excitation. The data were averaged over $1 \mathrm{ps}$ intervals around the indicated delays and are offset vertically for clarity. The high-q scattering $\left(q>0.2 \mathrm{~nm}^{-1}\right)$ from the chemically enriched regions shown in (a) is shaded grey. Illustrated between the two plots is the calculated local variation in the spin distribution $\mathrm{M}_{\mathrm{z}}$ within the $\mathrm{Gd}$ - and Fe-rich regions for $24 \mathrm{~mJ} / \mathrm{cm}^{2}$ pump fluence. 
a

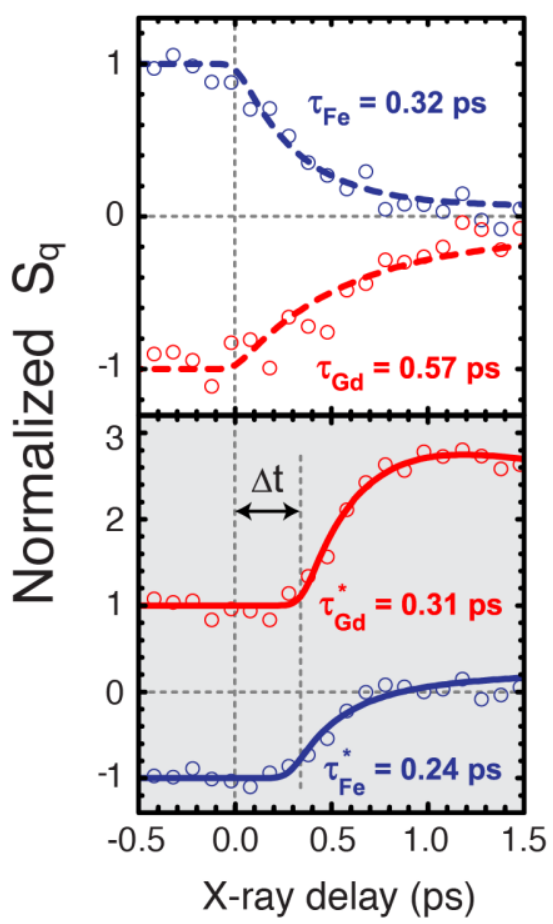

C

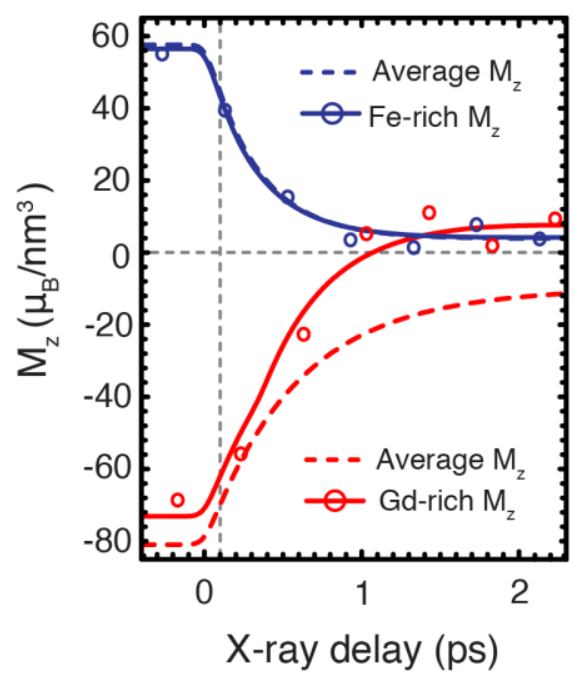

b

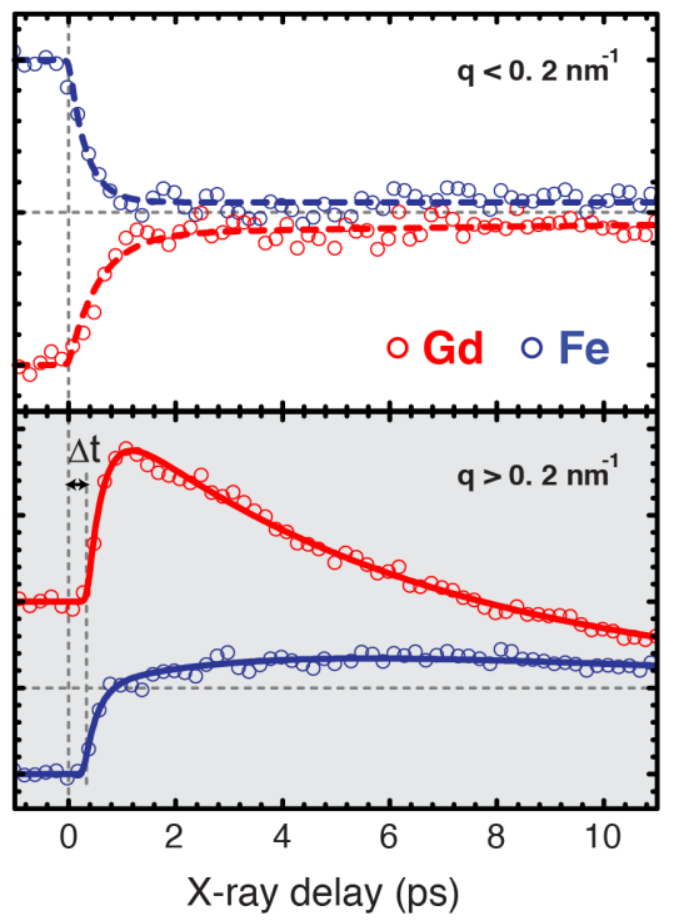

d

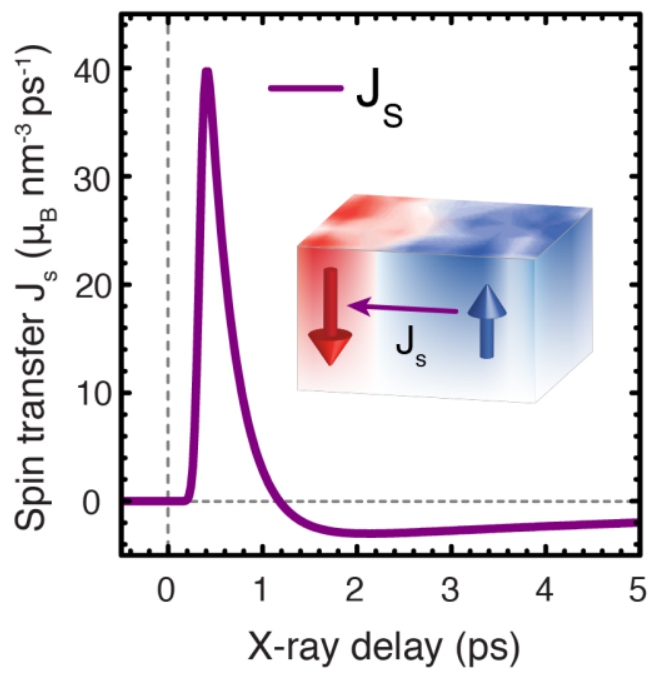

FIG. 3: Reversal of Gd spins within nanoscale Gd-rich regions as measured by resonant xray diffraction (a) Temporal evolution of magnetic diffraction, $S_{\mathrm{q}}$, for $\mathrm{Gd} 4 \mathrm{f}$ (red) and $\mathrm{Fe} 3 \mathrm{~d}$ (blue) spins measuring the local deviations from the average magnetization. (b) as in (a) but on a 10ps timescale. $S_{\mathrm{q}}$ signals were integrated over the low-q (top panel, $\mathrm{q}<0.2 \mathrm{~nm}^{-1}$ ) and gray shaded high-q (bottom panel, $\mathrm{q}>0.2 \mathrm{~nm}^{-1}$ ) regions of Figs. $2 \mathrm{~b}, \mathrm{c}$ and are normalized to the equilibrium values. Normalized $\left|S_{q}(t<0)\right|=1$ for both q-ranges and sublattices, although high-q $\mathrm{S}_{\mathrm{q}}$ for Gd is $\sim 4$ times greater than for Fe. Dashed and solid lines represent fits of exponential decay/rise functions to the data $(8,14,15)$. The observed delayed onset at $\Delta \mathrm{t}=360 \pm 50 \mathrm{fs}$ indicates that the nonlocal angular momentum transfer effectively occurs only after this time delay. The subsequent $\mathrm{Gd}$ and Fe rise times of $310 \pm 100$ and $240 \pm 100 \mathrm{fs}$, respectively, are essentially 
identical. Beyond 1ps, the $\mathrm{Gd}(\mathrm{Fe})$ data are well described by an exponential decay (rise) with a time constant of $6 \pm 2 \mathrm{ps}$. The Fe data is fit with an additional decay with time constant 8ps. (c) Time-evolution of $\mathrm{M}_{\mathrm{z}}$ in the Gd-rich and Fe-rich regions compared to the sample average. Solid lines are calculated from the fits in (a), while data points are averaged from data in (a). (d) Timeresolved angular momentum flow, $\mathrm{J}_{\mathrm{s}}$, into the $\mathrm{Gd}$-rich regions. The plot shows a time-delayed spike in the angular momentum transfer to the Gd spins in the Gd-rich nanoregions which lasts 1ps. Following this spin transfer to the Gd-rich nanoregions, a slower and weaker dissipation of spin from the region occurs. 